\title{
The Landau gauge gluon propagator in 4D SU(2) lattice gauge theory revisited: Gribov copies and scaling properties
}

\author{
I. L. Bogolubsky ${ }^{* a}$, E.-M. Ilgenfritz ${ }^{b}$, M. Müller-Preussker ${ }^{b}$, and A. Sternbeck ${ }^{c}$ \\ a Joint Institute for Nuclear Research, 141980 Dubna, Russia \\ ${ }^{b}$ Humboldt Universität zu Berlin, Institut für Physik, 12489 Berlin, Germany \\ ${ }^{c}$ CSSM, School of Chemistry \& Physics, University of Adelaide, SA 5005, Australia \\ E-mail: bogolubs@jinr.ru, ilgenfri@physik.hu-berlin.de, \\ mmpephysik.hu-berlin.de, andre.sternbeck@physik.uni-regensburg.de
}

Lattice results for the gluon propagator in $S U(2)$ pure gauge theory obtained on large lattices are presented. Simulated annealing is used throughout to fix the Landau gauge. We concentrate on checks for Gribov copy effects and for scaling properties. Our findings are similar to the ones in the $S U(3)$ case, supporting the decoupling-type infrared behaviour of the gluon propagator.

The XXVII International Symposium on Lattice Field Theory - LAT2009

July 26-31 2009

Peking University, Beijing, China

\footnotetext{
* Speaker.
} 


\section{Introduction}

Over the last years interesting results in Landau gauge gluodynamics have been found for gluon and ghost propagators (and, consequently, for the running coupling) both within the semianalytical Dyson-Schwinger(DS) as well as Functional Renormalization Group (FRG) approaches $[1,2]$ and with the help of lattice computations $[3,4,5,6,7]$. They have excited controversal debates about the behaviour in the deep infrared (IR) region. Within the DS and FRG approach it was demonstrated [2] that the behaviour strongly depends on the choice of the infrared limit of the ghost dressing function taken as a boundary condition for solving the (truncated) system of equations. The so-called scaling solution exhibits an IR singular well-defined power-like behaviour of the ghost dressing function and correspondingly a vanishing gluon propagator in agreement with the quite attractive confinement scenarios invented some time ago by Gribov and Zwanziger on one hand and by Kugo and Ojima on the other. Moreover, it was in accordance with BRST invariance properties.

Lattice results - as long as they are based on the assumption that one has to choose Gribov gauge copies as close as possible to the fundamental modular region - support with convincing numerical evidence the so-called decoupling solution with finite IR limits of both the gluon propagator and the ghost dressing function. Revisiting the case of $S U(2)$ lattice gauge theory we give here further evidence for this observation by a consequent use of an efficient gauge fixing method, the simulated annealing algorithm. Since previous lattice investigations of the IR limit both in $S U(2)$ as well as in $S U(3)$ were carried out at quite strong bare coupling values in order to reach largest possible physical volumes the continuum limit was not really under control. Therefore, in the given contribution to LATTICE '09 we have a look into the scaling properties of the gluon propagator, which seem to be a bit more involved in the $S U(2)$ than in the $S U(3)$ case. We neglect so-called $S U(2)$-flips which enlarge the class of Landau gauge orbits and allow to extremize the gauge functional even further.

\section{Landau gauge fixing, Gribov ambiguity and gluon propagator}

In order to fix the gauge on the lattice we apply gauge transformations $g(x) \in G=S U\left(N_{c}\right)$, $\left(N_{c}=2,3\right)$ to sets $\left\{U_{x, \mu}\right\}$ of link variables by mapping $U_{x, \mu} \rightarrow{ }^{g} U_{x, \mu}=g(x) U_{x, \mu} g^{\dagger}(x+\hat{\mu})$. The set of all admissible $\left\{{ }^{g} U_{x, \mu}\right\}$ for a given field $\left\{U_{x, \mu}\right\}$ is called a gauge orbit. The Landau gauge $\partial_{\mu} A_{\mu}=0$ for $A_{x+\hat{\mu} / 2, \mu}=\left(1 / 2 \operatorname{iag}_{0}\right)\left(U_{x, \mu}-U_{x, \mu}^{\dagger}\right)_{\text {traceless }}$ is fixed by searching for the local maxima $g_{\operatorname{lmx}}(x)$ of the gauge functional

$$
F_{U}[g]=\frac{1}{N_{c}} \sum_{x, \mu} \mathfrak{R e} \operatorname{Tr}^{g} U_{x, \mu} .
$$

In general for non-Abelian groups $G$ more than one local maximum $g_{\operatorname{lmx}}(x)$ can be found, the so-called Gribov copies. Since the values of the gauge functional $F_{U}[g]$ and other gauge-variant quantities $O\left({ }^{g} U\right)$ computed for various Gribov copies typically are correlated, further clarification of the gauge fixing condition is required. In case of Landau (or Coulomb) gauges for $S U\left(N_{c}\right)$ gauge theories it was proposed [8] to choose the global maximum $g_{g m x}(x)$ among all local ones, thus introducing the fundamental modular region (FMR) inside the Gribov region, the latter defined 
by the positivity region of the Faddeev-Popov operator [8]. In the present study we still keep the FMR condition. In practice it is hard - if not even impossible - to reach the FMR. Therefore, one is interested to improve the gauge fixing method and/or to apply the method of choice many times starting from random gauges in order to find the "best copies" closest to the FMR.

In what follows we reconsider the question of how the gluon propagator depends on the Gribov ambiguity when the (Landau) gauge is fixed on a lattice. This question has been addressed for relatively small lattice volumes already in preceding publications (e.g. in $[9,10,11])$. Note that in previous papers, instead of a comparison between two different gauge-fixing techniques, a comparison is made of the "best copy" (bc) with respect to the maximal $F_{U}[g]$ value achieved and "first copy" (fc), i.e. corresponding to a randomly chosen copy [10, 12]. In [11] "worst" copies were used for comparison, as well. There a visible Gribov copy effect was reported for the gluon propagator in the infrared. However, the effect appeared to be much more pronounced, when (i) the gauge orbit was extended by admitting nonperiodic (periodic up to $Z\left(N_{c}\right)$ ) gauge transformations (flips) and when (ii) the standard overrelaxation method (OR) was replaced by a simulated annealing algorithm (SA) - always followed by finalizing OR steps [13, 14, 15]. Moreover, the more efficient gauge fixing approach (SA + flips) led to a suppression of finite-volume effects, and indications were found that the influence of flip transformations on the gluon propagator gradually weakens with increasing linear lattice size $L$. But all these results were obtained at rather small lattice volumes.

In the present study for $S U(2)$ we neglect the flip gauge transformations and compare the SA method with the OR algorithm for strictly periodic gauge transformations. Our implementation of SA gauge fixing in the $S U(2)$ case differs from that for $S U(3)$ gluodynamics $[6,7]$ only in some technical details. We note that the "temperature" $T_{\max }$, from which SA cooling starts in the $S U(2)$ case, is chosen to be the "critical" value $T_{c r}$ of some phase transformation [16, 7], which takes place in the gauge-fixing field $g(x)$ interacting with the Monte Carlo generated equilibrium field $U_{x, \mu}$ according to the gauge functional (2.1). For the $S U(2)$ case it looks like a higher order phase transition [16] or even like a "crossover". Anyhow, we have chosen $T_{\max }=T_{c r}=1.1 \mathrm{in}$ most cases. Simulated annealing, also known as "stochastic optimization method" [17], in principle allows getting arbitrarily close to the global maximum $F_{U}\left[g_{g m x}\right]$, if the number of SA "cooling steps" is large enough. This is the underlying idea of our "single copy" method successfully used in our $S U(3)$ papers [6, 7]. In fact, in our $S U(2)$ computations we have used long SA chains with $O\left(10^{4}\right)$ steps from $T_{\max }$ down to $T_{\min }=0.01$ [6].

The comparison for the unrenormalized gluon propagator obtained with SA versus OR techniques on $80^{4}$ lattices at $\beta=2.30$ is shown in Fig. 1, where we have plotted only results for momenta $q^{2}$ surviving the so-called cylinder cut $[18,5]$. One can clearly see a noticeable difference between SA and OR gluon propagator values in the deep infrared region $q^{2}<0.2 \mathrm{GeV}^{2}$, where the Gribov effect leads to a qualitative change of the behaviour of the gluon propagator. The question remains, whether the Gribov copy effect for the gluon propagator weakens with a further increase of the lattice volume.

\section{Check of scaling and multiplicative renormalizability}

The progress to reach the infrared regime had the price to consider lattice gauge fields on 




Figure 1: Comparison of the unrenormalized gluon propagator obtained with SA and OR gauge fixing methods. The OR data are taken from Ref. [5].

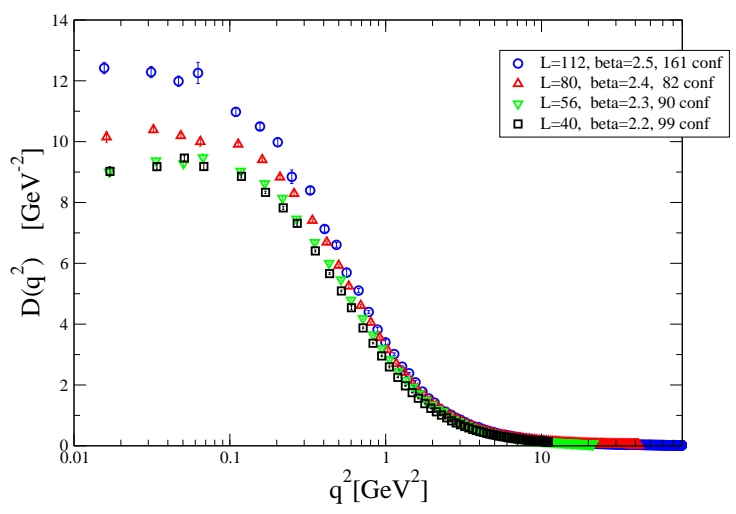

Figure 2: The unrenormalized gluon propagator $D\left(q^{2}\right)$ for various $(L, \beta)$ pairs, i.e. at fixed physical volume.

rather coarse lattices. To our knowledge the continuum limit expressed in a proper scaling and a multiplicative renormalization behaviour was not yet considered in detail on large volumes. We have made a step into this direction again neglecting the influence of $Z(2)$ flip transformations.

We have computed the gluon propagator on a sequence of lattices with increasing linear lattice size $L$ and $\beta$, choosing $(L, \beta)$ such that the physical volume was kept more or less constant. We have produced equilibrium ensembles of MC configurations and fixed the Landau gauge with the (single-copy) SA method for: $(L, \beta)=(40,2.2),(56,2.3),(80,2.4),(112,2.5)$, i.e. for a physical box size of approximately $10 \mathrm{fm}$. The bare gluon propagator $D\left(q^{2}\right)$ for these pairs of parameters is shown in Fig. 2.

There are quite strong differences which have not been observed in the $S U$ (3) case before. But 


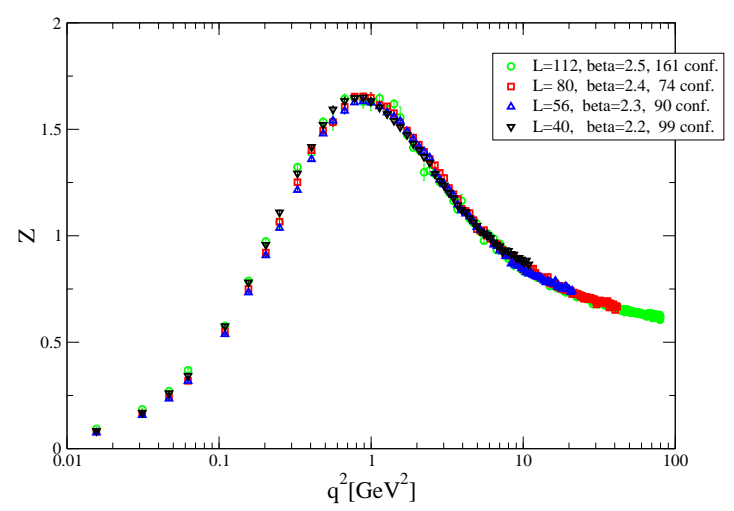

Figure 3: The renormalized gluon dressing function in the MOM scheme.

this does not come unexpected. In the continuum or scaling limit the bare propagator is expected to be multiplicative renormalizable as in perturbation theory. This means that between the results obtained at different lattice cutoffs a finite multiplicative renormalization up to lattice artifacts should be possible. In a finite volume - unavoidable for any lattice results - the multiplicative renormalization could be violated by finite-size effects.

In accordance with the standard momentum-subtraction $(\mathrm{MOM})$ renormalization scheme we have multiplicatively rescaled the bare gluon dressing functions $Z\left(q^{2}, \beta\right) \equiv q^{2} D\left(q^{2}\right)$ (for all $\beta$ values considered) equating the renormalized values at some $q=\mu$ to the tree-level value $Z_{\text {ren }}\left(\mu^{2}\right)=1$. The renormalization point was chosen at $\mu^{2}=5.8 \mathrm{GeV}^{2}$, sufficiently far away from the cutoff momentum $q_{\max }^{2}=79.4 \mathrm{GeV}^{2}$ for $\beta=2.50$. For illustration the finite renormalization factors $Z\left(\mu^{2}, \beta\right) / Z\left(\mu^{2}, \beta=2.5\right)$ are shown in the Table 1 . They were obtained by interpolating between the 7 data points closest to the chosen scale $\mu$ for $\beta=2.5$.

Table 1: Finite renormalization factors for $\mu^{2}=5.8 \mathrm{GeV}^{2}$.

\begin{tabular}{|c|c|}
\hline$\beta$ & $Z\left(\mu^{2}, \beta\right) / Z\left(\mu^{2}, \beta=2.5\right)$ \\
\hline 2.2 & 0.815 \\
2.3 & 0.8925 \\
2.4 & 0.9489 \\
\hline
\end{tabular}

The MOM-renormalized dressing function is plotted in Fig. 3. One clearly sees that the three curves for the renormalized dressing function $Z_{r e n}\left(q^{2}\right)$ obtained at lattice sizes $L=56,80,112$ lie nicely on top of each other, thus confirming the expected multiplicative renormalizability. For $\beta=$ 2.2 and $L=40$ there are some scaling violations that can be understood as lattice artifacts. Some slight variations of the curve obtained with the largest lattice $L=112$ probably can be attributed to problems with the still unsufficient Monte Carlo statistics and/or autocorrelations.

\section{Conclusions}

The comparison of OR- and SA-based results for the gluon propagator for $L=80$ and $\beta=2.3$ 
clearly shows a noticeable Gribov copy effect in the range of momenta $q^{2}<0.2 \mathrm{GeV}^{2}$. At the moment the (dis)appearance of this effect for even larger volumes is an interesting open problem. An open question is also, whether our results obtained on large volumes will be modified, when $Z$ (2) flips are taken into account. This is a matter of research in a forthcoming papers of one of the coauthors [19].

Using SA-based Landau gauge fixing we have got numerical confirmation for a nonperturbative multiplicative renormalizability for the gluon dressing function. Finite-size effects already seem to be negligible in the given range of momenta.

\section{References}

[1] Ph. Boucaud, J.P. Leroy, A. Le Yaouanc, J. Micheli, O. Pene, J. Rodriguez-Quintero, JHEP0806 (2008) 012, arXiv:0801.2721; JHEP0806 (2008) 099, arXiv:0803.2161.

[2] C.S. Fischer, A. Maas, J.M.Pawlowski, Annals Phys. 324 (2009) 2408, arXiv:0810.1987.

[3] A. Cucchieri, T. Mendes, PoS LAT2007 (2007) 297, arXiv:0710.0412.

[4] A. Cucchieri, T. Mendes, Phys. Rev. Lett. 100 (2008) 241601, arXiv:0712.3517.

[5] A. Sternbeck, L. von Smekal, D.B. Leinweber, A.G. Williams, PoS LAT2007 (2007) 340, arXiv:0710.1982.

[6] I.L. Bogolubsky, E.-M. Ilgenfritz, M. Müller-Preussker, A. Sternbeck, PoS LAT2007 (2007) 290, arXiv:0710.1968.

[7] I.L. Bogolubsky, E.-M. Ilgenfritz, M. Müller-Preussker, A. Sternbeck, Phys. Lett. B676 (2009) 69, arXiv:0901.0736.

[8] D. Zwanziger, Nucl. Phys. B412 (1994), 657.

[9] A.Cucchieri, Nucl. Phys. B508 (1997) 353, arXiv:hep-lat/9705005.

[10] T.D. Bakeev, E.-M. Ilgenfritz, V.K. Mitrjushkin, M. Müller-Preussker, Phys.Rev. D69 (2004) 074507, arXiv:hep-lat/0311041.

[11] P. J. Silva, O. Oliveira, Nucl. Phys. B690 (2004) 177, arXiv:hep-lat/0403026.

[12] A. Sternbeck, E.-M. Ilgenfritz, M. Müller-Preussker, A. Schiller, Nucl.Phys.Proc.Suppl. 140 (2005) 653, arXiv:hep-lat/0409125; AIP Conf. Proc. 756 (2005) 284, arXiv:hep-lat/0412011.

[13] I. L. Bogolubsky, G. Burgio, V. K. Mitrjushkin, M. Müller-Preussker, Phys. Rev. D74 (2006), 034503, arXiv:hep-lat/0511056.

[14] I. L. Bogolubsky, V. G. Bornyakov, G. Burgio, E.-M. Ilgenfritz, M. Müller-Preussker, V. K. Mitrjushkin, Phys. Rev. D77 (2008) 014504, arXiv:0707.3611.

[15] V. G. Bornyakov, V. K. Mitrjushkin, M. Müller-Preussker, Phys. Rev. D79 (2009) 074504, arXiv:0812.2761.

[16] P.Schemel, diploma thesis, Humboldt University.

[17] S. Kirkpatrick, C. D. Gelatt Jr., and M. P. Vecchi, Science 220 (1983) 671; V. Cerny, J. Opt. The. Appl. 45 (1985) 41.

[18] D.B.Leinweber, J.I.Skullerud, A.G.Williams, C.Parrinello, Phys. Rev. D60 (1999) 094507, arXiv:hep-lat/9811027.

[19] V. G. Bornyakov, V. K. Mitrjushkin, M. Müller-Preussker, work in progress. 